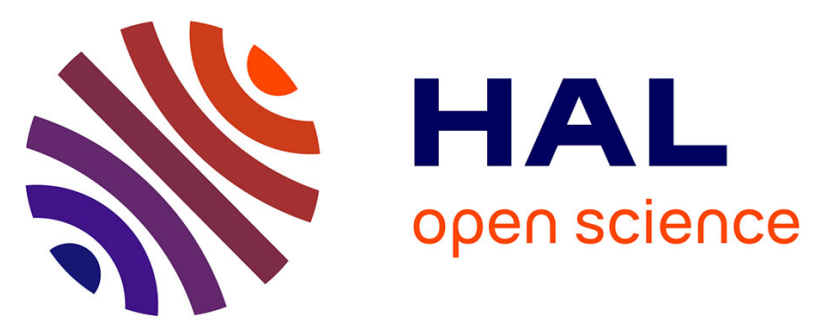

\title{
Synthesis of picosecond pulses by spectral compression and shaping of femtosecond pulses in engineered quadratic nonlinear media
}

\author{
M. Marangoni, D. Brida, Matteo Conforti, A. D Capobianco, C. Manzoni, F. \\ Baronio, G. F Nalesso, C. de Angelis, Roberta Ramponi, G. Cerullo
}

\section{To cite this version:}

M. Marangoni, D. Brida, Matteo Conforti, A. D Capobianco, C. Manzoni, et al.. Synthesis of picosecond pulses by spectral compression and shaping of femtosecond pulses in engineered quadratic nonlinear media. Optics Letters, 2009, 34 (3), pp.241. 10.1364/OL.34.000241 . hal-02397636

\section{HAL Id: hal-02397636 https://hal.science/hal-02397636}

Submitted on 6 Dec 2019

HAL is a multi-disciplinary open access archive for the deposit and dissemination of scientific research documents, whether they are published or not. The documents may come from teaching and research institutions in France or abroad, or from public or private research centers.
L'archive ouverte pluridisciplinaire HAL, est destinée au dépôt et à la diffusion de documents scientifiques de niveau recherche, publiés ou non, émanant des établissements d'enseignement et de recherche français ou étrangers, des laboratoires publics ou privés. 


\title{
Synthesis of Picosecond Pulses by Spectral Compression and Shaping
}

\section{of Femtosecond Pulses in Engineered Quadratic Nonlinear Media}

\author{
M. Marangoni ${ }^{1 *}$, D. Brida ${ }^{1}$, M. Conforti ${ }^{2}$, A. D. Capobianco ${ }^{3}$, C. Manzoni ${ }^{1}$, \\ F. Baronio ${ }^{2}$, G. F. Nalesso ${ }^{3}$, C. De Angelis ${ }^{2}$, R. Ramponi ${ }^{1}$, and G. Cerullo ${ }^{1}$ \\ ${ }^{1}$ National Laboratory for Ultrafast and Ultraintense Optical Science, INFM-CNR, \\ Dipartimento di Fisica - Politecnico di Milano, Piazza Leonardo da Vinci 32, 20133 Milano, Italy \\ ${ }^{2}$ Dipartimento di Elettronica per l'Automazione, Università di Brescia, via Branze 38, 25123 \\ Brescia, Italy \\ ${ }^{3}$ Dipartimento di Ingegneria dell'Informazione - Università degli Studi di Padova, \\ Via Gradenigo 6/B, 35131 Padova, Italy \\ *Corresponding author: marco.marangoni@polimi.it
}

\begin{abstract}
Narrow-bandwidth picosecond pulses of predetermined spectral and temporal shape are generated with high efficiency by frequency conversion of femtosecond pulses in lithium tantalate crystals with engineered quasi-phase-matching structures. We give examples of the synthesis of gaussian and supergaussian picosecond pulses and also of a pair of synchronized phase-coherent picosecond pulses with a given carrier-frequency difference.
\end{abstract}

OCIS codes: (190.4410) Nonlinear optics, parametric processes; (190.7110) Ultrafast nonlinear optics; (320.5390) Picosecond phenomena. 
Several spectroscopic techniques require the synchronization between independently tunable narrowband picosecond pulses or between picosecond and femtosecond pulses. A representative example is Coherent Anti-Stokes Raman Scattering (CARS), which enables label-free molecular identification [1]: depending on the configuration it can require either two narrowband picosecond pulses [2], or the combination of a picosecond and a femtosecond pulse [3]. A second example is femtosecond stimulated Raman spectroscopy [4,5], which allows visualization of the structural dynamics in a photoexcited molecule and requires an actinic femtosecond excitation pulse combined with a picosecond pump pulse and a femtosecond probe pulse for driving the Raman process.

The most common sources of tunable picosecond pulses are optical parametric oscillators (OPOs), which provide high average power. OPOs suffer however from a costly and complex architecture requiring the synchronization of two optical cavities, and, moreover, they can not be used in experiments requiring mixing of picosecond and femtosecond pulses. On the other hand femtosecond sources are becoming more and more compact and reliable. For this reason several linear and nonlinear techniques have been proposed for the synthesis of tunable narrowband picosecond pulses starting from femtosecond pulses. The simplest approach is the spectral filtering of a femtosecond pulse with either a bandpass filter [6] or the combination of a slit with a $4 \mathrm{f}$ spectral shaper [4]; its main drawback is to introduce a strong power penalty, by an amount equal at least to the ratio between input and filtered bandwidth. Techniques based on self-phase-modulation processes in photonic crystal fibers in the presence of negatively chirped input pulses have proved to be useful for compression ratios as high as 10 , at the price, however, of poor power scalability and stability [7]. Second order nonlinear processes driven by chirped pulses have also been exploited, using for example sum-frequency-generation (SFG) between pulses with opposite chirp [8] or difference-frequency-generation between pulses with the same chirp [9]. These techniques 
are however experimentally challenging, since they require the use of a pair of carefully balanced gratings (or prisms) pulse stretchers/compressors.

Recent papers $[10,11]$ showed that a second-harmonic-generation (SHG) process in the presence of high group-velocity-mismatch (GVM) between the fundamental frequency (FF) and the second harmonic (SH) pulse can lead to the efficient generation of narrowband $\mathrm{SH}$ pulses starting from broadband FF ones. This result can be understood by considering that, in addition to the SHG process, there is also an efficient SFG process between spectral components of the FF that are symmetric with respect to the SHG phase-matching frequency, allowing efficient energy transfer from the broadband FF pulse to the narrowband $\mathrm{SH}$ one. By using periodically-poled materials, which exhibit very high GVM and nonlinearity, spectral compression ratios as high as 100 , tunability over $1600 \mathrm{~cm}^{-1}$ and conversion efficiencies of $20 \%$ have been experimentally demonstrated [11]. The main limitation of this approach was the difficulty in controlling the spectral and the temporal shape of the generated pulses.

In this paper we demonstrate how to properly engineer quasi-phase-matching (QPM) structures in order to generate with high efficiency SH pulses with transform-limited picosecond duration, predetermined spectral and temporal shape, and synchronization with femtosecond pulses. As representative examples we report the synthesis of gaussian and super-gaussian SH pulses. We also show how QPM structures can be used for the synthesis of a pair of phase-coherent picosecond super-gaussian pulses with a given carrier-frequency detuning. Experimental results are in excellent agreement with numerical calculations.

The starting point of our work is a method recently proposed for the design of non-uniform QPM gratings, allowing control of both amplitude and phase of the SH pulses in conditions of strong FF depletion [12]. Such method overcomes the limitations of other approaches [13, 14], that were valid only for low FF depletion. We used the method to design three different gratings aimed at the 
synthesis of the following SH target pulses: a) gaussian pulse , b) supergaussian pulse, c) pair of two picosecond phase-coherent supergaussian pulses with a given carrier-frequency offset. The design algorithm, which makes no simplifying assumptions as to group-velocity-dispersion and pump depletion, allows for the synthesis of the nonlinearity profile that provides, for a given FF pulse, the SH pulse that best matches the target one.

Figure 1 reports the results obtained in the case of the gaussian target pulse with 2 ps FWHM duration. We use a $2.5 \mathrm{~cm}$ long stoichiometric lithium tantalate (SLT) crystal (nonlinear coefficient d33=10.7 pm/V), a 40 fs long FF gaussian pulse with $1400 \mathrm{~nm}$ carrier wavelength, a peak intensity of $10 \mathrm{GW} / \mathrm{cm}^{2}$ and a group delay dispersion (GDD) of $-1300 \mathrm{fs}^{2}$, which corresponds, with the opposite sign, to half that introduced by the crystal. Panel (a) reports the calculated behaviour of both the real and imaginary part of the effective nonlinearity as a function of the propagation coordinate $z$ : it exhibits a smooth longitudinal variation from zero to its maximum value $\chi_{0} / \pi$ $\left(\chi_{0}=\omega \mathrm{d}_{\mathrm{eff}} / \mathrm{nc}\right)$, required to obtain a clean spectral shaping without ringing effects due to abrupt changes of the nonlinearity. This profile is achieved with a second-order QPM grating, which allows for a continuous variation of the effective nonlinear coefficient from zero to its maximum value by changing the duty cycle from 50:50 to 75:25. This point is highlighted in panel (b), where the domain dimensions required to implement the nonlinearity profile are reported. Note that a firstorder grating would provide a maximum nonlinearity which is a factor of two higher than the second-order one [15]; this is however not needed since, with the available intensities, the FF pulse can be easily depleted. On the other hand, it would require shrinking to zero the ferroelectric domain size in order to achieve small nonlinearities, with obvious technological limitations. Panel (c) shows the calculated spectral shape of the SH pulse (solid line) as compared to the FF input spectrum (dashed line). The spectral compression factor is as high as 100 , which is a remarkable value if one considers an expected conversion efficiency of $45 \%$. Panel (d) reports the calculated 
temporal shape of the SH pulse at the crystal output for the designed non-uniform QPM grating depicted in panel (b).

Figure 2 reports the results for the synthesis of two picosecond super-gaussian pulses with 3 ps duration and frequency difference of $200 \mathrm{~cm}^{-1}$. The effective nonlinearity is again zero at the crystal ends, where the duty-cycle is 50:50. The central part of the nonlinearity profile is now enveloped by a nearly flat-top apodization profile needed for the synthesis of the super-gaussian pulses, with a pronounced bump towards the crystal end in order to compensate for pump depletion. The oscillating behaviour is due to the beating between the two periods needed for the synthesis of the two colours. The SH spectrum presents two well separated peaks around 690 and $710 \mathrm{~nm}$ (corresponding to the target frequency difference of $200 \mathrm{~cm}^{-1}$ ) without any side-lobes thanks to the apodization profile. In the temporal domain the two $\mathrm{SH}$ pulses are superimposed and, since originating from the same FF pulse, they are also phase-coherent, which results in high visibility beating fringes in the (d) panel. The envelope of such profile presents the expected flat-top supergaussian shape.

The experiments were performed using an optical parametric amplifier pumped by a $1-\mathrm{kHz}$ amplified Ti:sapphire system and delivering 40-50 fs long pulses with energy up to $4 \mu \mathrm{J}$ and tunability from 1.2 to $1.8 \mu \mathrm{m}$. Such pulses are pre-chirped by a pair of Brewster-cut LaFN28 prisms at a $32.5-\mathrm{cm}$ distance so as to acquire the required negative GDD. They are then focused on the crystal input face with a spot size of $213 \mu \mathrm{m}$. Several 1-mm-thick 2.5-cm-long crystals with QPM gratings corresponding to the above described target $\mathrm{SH}$ pulses were manufactured by $\mathrm{HC}$ Photonics. The SH spectra are recorded either by an optical spectrum analyzer with $0.01 \mathrm{~nm}$ spectral resolution or by a spectrometer with $0.1 \mathrm{~nm}$ resolution. The temporal profile of the $\mathrm{SH}$ pulses is characterized by cross-correlation with a fraction of the 50 -fs FF pulses emitted by the Ti:sapphire system. 
Figure 3 reports for each target pulse the spectrum (solid line on the left) and the crosscorrelation (solid line on the right) of the $\mathrm{SH}$ pulses at the output of the crystal. For the gaussian and super-gaussian pulses, the measured spectra exhibit some slight deviations from the expected ones with respect to the shape and a very good agreement with respect to the FWHM spectral widths, respectively equal to 0.5 and $0.2 \mathrm{~nm}\left(10\right.$ and $\left.4 \mathrm{~cm}^{-1}\right)$. In the temporal domain the crosscorrelation traces can be very well fitted by the Gaussian and super-gaussian functions reported with bold lines in the figure. Their FWHM values are respectively equal to 2.8 and 5 ps, which compare well with the expected 2 and 5 ps target values. The presence of some satellites in the leading and trailing edges of the traces can be ascribed to slight deviations from the 50:50 duty cycle at the crystal ends, where the expected nonlinearity is zero. The conversion efficiencies for a pulse energy of $150 \mathrm{~nJ}$ were 17 and $20 \%$ in the two cases respectively.

Fig. 3 also shows the results for the synthesis of a pair of picosecond pulses, with frequency offsets of $200 \mathrm{~cm}^{-1}$ and $400 \mathrm{~cm}^{-1}$ respectively. In both cases, two distinct peaks appear at the expected wavelengths, each of them with a FWHM of $0.56 \mathrm{~nm}$. The temporal profile, reported for the $200 \mathrm{~cm}^{-1}$ case, is in excellent agreement with the numerical one, both in terms of the oscillating period, and of the high contrast between the beating fringes, which is only limited by the 50 fs duration of the gating pulse. This beating is the signature of the very high phase coherence between the two colours, which are synthesized starting from a single pulse.

In conclusion, engineered QPM structures have been proved suitable for the efficient synthesis of picosecond pulses with arbitrary waveforms starting from femtosecond pulses. Thanks to the apodization profile of the nonlinearity, the typical side-lobes that are found with uniform QPM structures can be eliminated, which is a crucial advantage for achieving high spectral resolution in spectroscopic applications. The simultaneous synthesis of two narrowband, synchronized, and 
phase coherent pulses with arbitrary frequency difference is also demonstrated, paving the way to a compact single-beam CARS spectrometer. 


\section{REFERENCES IN OPTICS LETTERS FORMAT}

1. C.L. Evans and X. S. Xie, Annu. Rev. Anal. Chem. 1, 883 (2008).

2. E. O. Potma, D. J. Jones, J. Cheng, X. S. Xie, and J. Ye, Opt. Lett. 27, 1168 (2002).

3. D. Pestov, R. K. Murawski, G. O. Ariunbold, X. Wang, M. Zhi, A. V. Sokolov, V. A. Sautenkov, Y. V. Rostovtsev, A. Dogariu, Y. Huang, M. O. Scully, Science 316, 265 (2007).

4. D. W. McCamant, P. Kukura, and R. A. Mathies, Rev. Sci. Instrum. 75, 4971 (2004).

5. P. Kukura, D. W. McCamant, S. Yoon, D. B. Wandschneider, and R. A. Mathies, Science 310, $1006(2005)$.

6. A. N. Bordenyuk, H. Jayathilake, and A. V. Benderskii, J. Phys. Chem. B 109, 15941 (2005).

7. E. R. Andresen, J. Thøgersen, and S. R. Keiding, Opt. Lett. 30, 2025 (2005).

8. F. Raoult, A. C. L. Boscheron, D. Husson, C. Sauteret, A. Modena, V. Malka, F. Dorchies, and A. Migus, Opt. Lett. 23, 1117 (1998).

9. H. Luo, L. Qian, P. Yuan, and H. Zhu, Opt. Express 14, 10631 (2006).

10. K. Moutzouris, F. Adler, F. Sotier, D. Träutlein, and A. Leitenstorfer, Opt. Lett. 31, 1148 (2006).

11. M. Marangoni, D. Brida, M. Quintavalle, G. Cirmi, F. M. Pigozzo, C. Manzoni, F. Baronio, A. D. Capobianco, and G. Cerullo, Opt. Express 15, 8884 (2007).

12. M. Conforti, F. Baronio, and C. De Angelis, Opt. Lett. 32, 1779 (2007).

13. G. Imeshev, M. M. Fejer, A. Galvanauskas, and D. Harter, Opt. Lett. 26, 268 (2001).

14. Ł. Kornaszewski, M. Kohler, U. K. Sapaev, and D. T. Reid, Opt. Lett. 33, 378 (2008).

\section{5. reference QPM second'ordine}




\section{FULL REFERENCES}

1. C.L. Evans and X. S. Xie, "Coherent Anti-Stokes Raman Scattering Microscopy: Chemically Selective Imaging for Biology and Medicine,” Annu. Rev. Anal. Chem. 1, 883-909 (2008).

2. E. O. Potma, D. J. Jones, J. Cheng, X. S. Xie, and J. Ye, "High-sensitivity coherent anti-Stokes Raman scattering microscopy with two tightly synchronized picosecond lasers,” Opt. Lett. 27, $1168-1170(2002)$.

3. D. Pestov, R. K. Murawski, G. O. Ariunbold, X. Wang, M. Zhi, A. V. Sokolov, V. A. Sautenkov, Y. V. Rostovtsev, A. Dogariu, Y. Huang, M. O. Scully, “Optimizing the LaserPulse Configuration for Coherent Raman Spectroscopy,” Science 316, 265-268 (2007).

4. D. W. McCamant, P. Kukura, and R. A. Mathies, "Femtosecond broadband stimulated Raman spectroscopy: Apparatus and methods,” Rev. Sci. Instrum. 75, 4971-4980 (2004).

5. P. Kukura, D. W. McCamant, S. Yoon, D. B. Wandschneider, and R. A. Mathies, "Structural observation of the primary isomerization in vision with femtosecond-stimulated Raman," Science 310, 1006-1009 (2005).

6. A. N. Bordenyuk, H. Jayathilake, and A. V. Benderskii, "Coherent Vibrational Quantum Beats as a Probe of Langmuir-Blodgett Monolayers,” J. Phys. Chem. B 109, 15941-15949 (2005).

7. E. R. Andresen, J. Thøgersen, and S. R. Keiding, "Spectral compression of femtosecond pulses in photonic crystal fibers," Opt. Lett. 30, 2025-2027 (2005).

8. H. Luo, L. Qian, P. Yuan, and H. Zhu, "Generation of tunable narrowband pulses initiating from a femtosecond optical parametric amplifier,” Opt. Express 14, 10631-10635 (2006).

9. F. Raoult, A. C. L. Boscheron, D. Husson, C. Sauteret, A. Modena, V. Malka, F. Dorchies, and A. Migus, "Efficient generation of narrow-bandwidth picosecond pulses by frequency doubling of femtosecond chirped pulses," Opt. Lett. 23, 1117-1119 (1998). 
10. K. Moutzouris, F. Adler, F. Sotier, D. Träutlein, and A. Leitenstorfer, "Multimilliwatt ultrashort pulses continuously tunable in the visible from a compact fiber source", Opt. Lett. 31, 1148-1150 (2006).

11. M. Marangoni, D. Brida, M. Quintavalle, G. Cirmi, F. M. Pigozzo, C. Manzoni, F. Baronio, A. D. Capobianco, and G. Cerullo, "Narrow-bandwidth picosecond pulses by spectral compression of femtosecond pulses in second-order nonlinear crystals," Opt. Express 15, 88848891 (2007).

12. M. Conforti, F. Baronio, and C. De Angelis, "From femtosecond infrared to picosecond visible pulses: temporal shaping with high-efficiency conversion,” Opt. Lett. 32, 1779-1781 (2007).

13. G. Imeshev, M. M. Fejer, A. Galvanauskas, and D. Harter, "Generation of dual-wavelength pulses by frequency doubling with quasi-phase-matching gratings," Opt. Lett. 26, 268-270 (2001).

14. Ł. Kornaszewski, M. Kohler, U. K. Sapaev, and D. T. Reid, "Designer femtosecond pulse shaping using grating-engineered quasi-phase-matching in lithium niobate," Opt. Lett. 33, 378$380(2008)$.

\section{5. reference QPM second'ordine}




\section{FIGURE CAPTIONS}

FIG. 1. Synthesis of 2 ps long gaussian SH pulses at $\lambda=0.7 \mu \mathrm{m}$ from a 40 fs long FF pulses: (a) optimized nonlinearity profile normalized to $\chi_{\max }=2 / \pi \chi_{0}$; (b) minimum and maximum domain dimensions as a function of propagation distance, and, in the insets, enlarged view of the domain sequence; (c) spectra of the input FF pulse (dashed line) and of the output SH pulse; (d) temporal profile of the SH pulse at the output.

FIG. 2. Same sequence of panels as in Fig. 1 in the case of the generation of a pair of phasecoherent supergaussian pulses with a carrier frequency differing by $200 \mathrm{~cm}^{-1}$.

FIG. 3. Experimental spectrum and crosscorrelation of the SH pulses at the crystal output (solid lines) as compared to the calculated ones (dotted line) in the case of the synthesis of: (a) gaussian pulse; (b) supergaussian pulse; (c) pair of supergaussian pulses with a $200 \mathrm{~cm}^{-1}$ and $400 \mathrm{~cm}^{-1}$ frequency offset (the crosscorrelation refers to the $200 \mathrm{~cm}^{-1}$ case). 
M. Marangoni et al., "Synthesis of Picosecond Pulses ...."

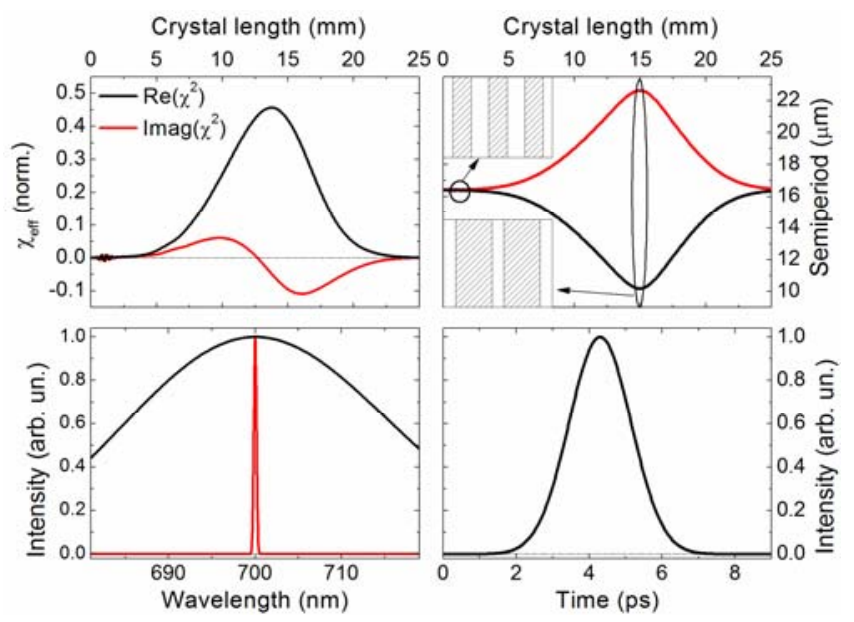

Figure 1 
M. Marangoni et al., "Synthesis of Picosecond Pulses ...."

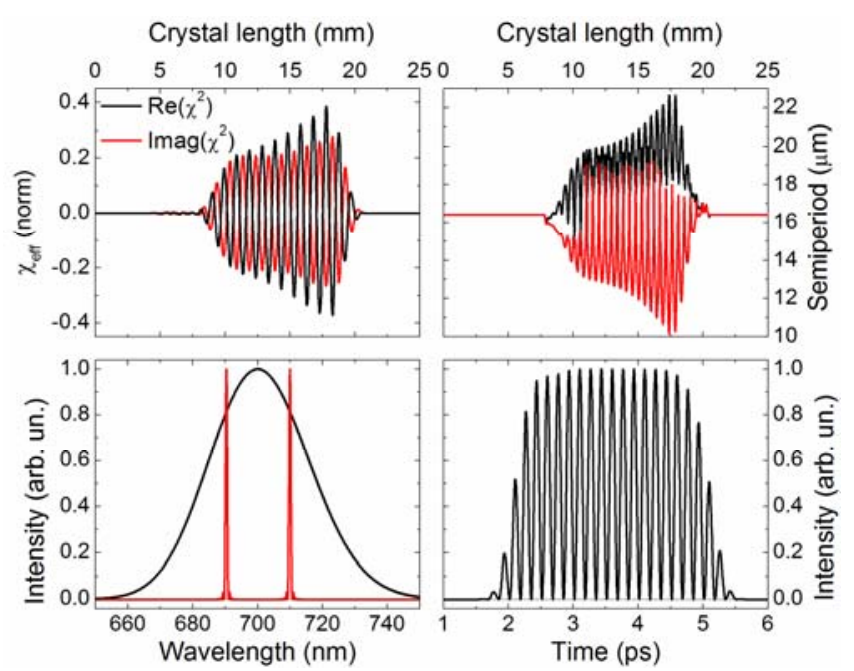

Figure 2 
M. Marangoni et al., "Synthesis of Picosecond Pulses ...."
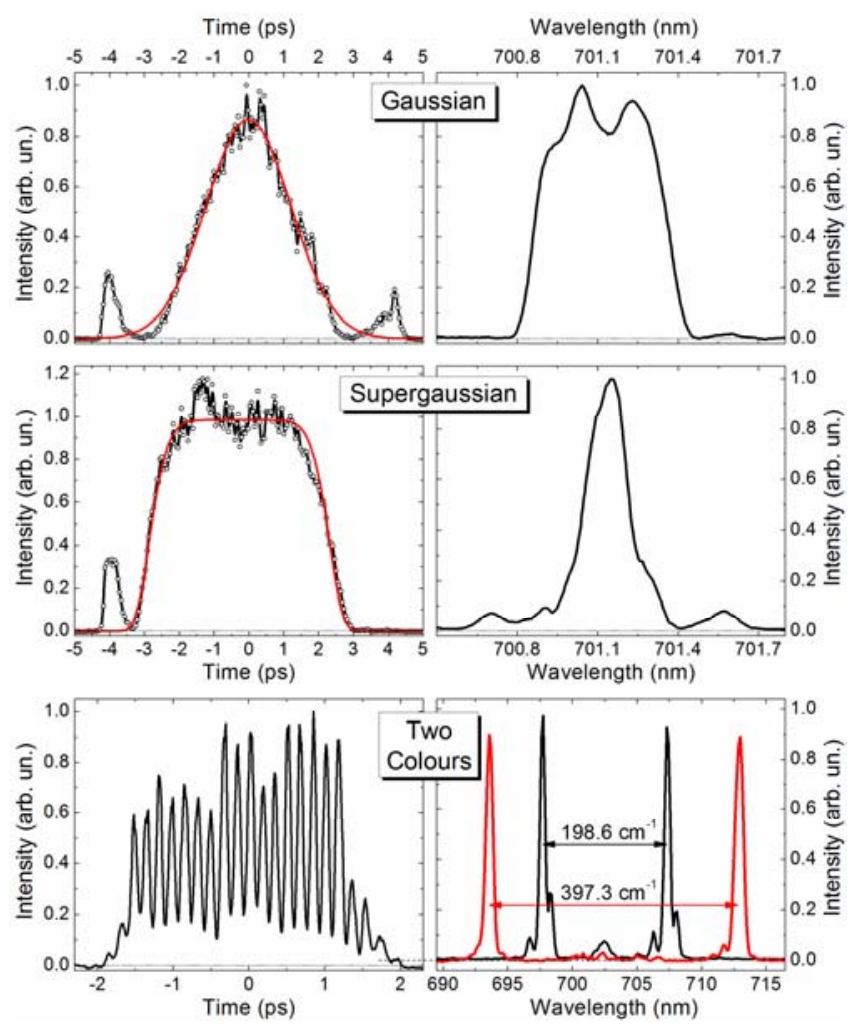

Figure 3 